\title{
Die Sprache der Weinkenner und ihre verschiedenen Aspekte
}

\author{
Gabriela RYKALOVÁ
}

\begin{abstract}
The lang uage of wine connoisseurs and its various aspects

The article introduces the language of wine connoisseurs in all its forms and, based on a number of specific examples from different genres and various types of texts (specialist literature, catalogues, wine labels, etc.), aims to stimulate discussion on the circumstances which influence the level of specialization in connection with the choice of linguistic resources.
\end{abstract}

Keywords: professional language, language of wine, language of wine connoisseurs, specialist area, style

DOI: doi.org/10.15452/StudiaGermanistica.2020.26.0005

\section{Einleitung}

Die Sprache als Handlungsinstrument dient der kommunikativen Tätigkeit in unterschiedlichen Lebensbereichen. Sei es im Alltag oder im beruflichen Leben: Die Sprache ist eines der wichtigsten Instrumente der Verständigung.

Die Sprache von Önologen, Weindegustatoren, Sommeliers, Winzern, Kellermeistern und anderen Fachleuten sowie Weinhändlern und Weinliebhabern, die als Weinkenner bezeichnet werden können, besteht auf der einen Seite aus Fachbegriffen, die durch Definitionen präzise charakterisiert werden, auf der anderen Seite aus Vergleichen und Metaphern, um den Geschmack, die Farbe und den Geruch von Weinen möglichst genau zu beschreiben und auch um auf den Konsumenten zu zielen und den Wein als Produkt möglichst effektiv zu vermarkten.

Wie vielfältig die Sprache über den Wein sein kann, zeigt Priewe in seinem Buch ,Wein. Die praktische Schule‘ (Priewe 2001):

\section{Weinsprache}

Die Weinsprache besitzt ein eigenes Vokabular, aber jeder Weintrinker neigt zu eigenen Vokabeln. Mit seinen Kommentaren zum Wein - egal, ob er damit Zustimmung oder Ablehnung ausdrücken will - outet er sich und demonstriert, zu welchem Typ er gehört.

Der brave Langweiler: gefällig, artig, vollmundig, sortentypisch, reintönig, blumig, rassig, rund, fest, nachhaltig, ansprechend.

Der „Weinrambo“: geiler Tropfen, Superstoff, sexy Wein, tolles Tannin, Wahnsinnsgeschmack, höllisches Holunderbouquet, Granatenwein, explosive Kraft, monumental, titanisch, gigantisch. 
Der einfallslose Experte: komplex. Vielschichtig, gute Struktur, mittlerer Körper, glatte Länge, gut eingebundene Säure.

Der Fabulierer: Am Gaumen ist die Hölle los. / Der Wein ist wie ein Schlag in die Magengrube. I Zart wie der Biss einer jungen Viper. / Duftet wie der Schweiß der Engel.

Der unnahbare Fachmann: körniges Tannin, ausgeglichene Adstringenz, feinsandige Textur, gelbe Früchte, oberflächliche Malolaktik, pferdige Terroir-Note.

Der kunstsinnige Fachmann: ... wie eine Fuge von Bach .../...üppig wie eine Figur von Rubens... /... heiter wie ein Menuett von Mozart... /...das Bouquet ist wie vom Winde verweht...

Der moderne „Weinsoftie“: lecker, echt köstlich, chic, richtig nett.

(Priewe 2001:69)

Der Beitrag setzt sich zum Ziel, die Sprache der Weinkenner mit all ihren Facetten vorzustellen und möchte anhand von konkreten Beispielen aus unterschiedlichen Textbelegen (Fachliteratur, Kataloge, Weinetiketten) die Diskussion anregen, ob es eine klare Abgrenzung zwischen der Sprache der Weinkenner als Fachsprache und der Sprache der Marketingexperten gibt.

\section{Das Untersuchungskorpus}

Das Untersuchungskorpus bilden drei Gruppen von Belegquellen: Lehrbücher, Sachbücher und Weinbeschreibungen als produktbegleitende Werbetexte. Die Texte unterscheiden sich hinsichtlich des Adressatenbezuges und der Funktion bzw. Intention des Autors/der Autoren.

In Anlehnung an die von Adamzik vorgestellte Typologie schriftlicher Fachtextsorten (vgl. 2018:261) handelt es sich um Texte fachexterner Kommunikation:

1. ,didaktisierende Textsorten':

- Vogt, Ernst/ Götz, Bruno: Weinbau. Ein Lehr- und Handbuch für Praxis und Schule, 1979.

- Vogt, Ernst / Jakob, Ludwig / Lemperle, Edmund / Weiss, Erich: Der Wein, 1984.

- Kadisch, Erwin: Der Winzer 1. Weinbau, 1986.

- Meidinger, Friedrich: Der Winzer 2. Kellerwirtschaft, 1989.

- Bergner, Karl-Gustav / Lemperle, Edmund: Weinkompendium. Botanik. Sorten. Anbau. Bereitung, 2001.

2. ,popularisierende Textsorten':

- Broadbent, Michael J.: Weine prüfen, kennen, geniessen, 1979.

- Gräter, Carlheinz: Frankens Reben im Portrait, 1990.

- Althaus, Hans Peter: Kleines Wörterbuch der Weinsprache, 2008.

- Robinson, Jancis: Weinexperte in 24 Stunden. Von der angesehensten Weinexpertin der Welt, 2017.

3. ,verhaltenssteuernde Textsorten":

- Webseiten: https://www.weingut-juliusspital.de

- Jacques' Neuvorstellungen. April 2019

- Sommerzeit ist Weinzeit

\section{Fach als Gegenstandsbereich}

Über welches Fach wird aber im Zusammenhang mit dem Wein gesprochen?

Wird über Fachtexte und Fachsprache gesprochen, sollte erklärt werden, was unter dem Begriff „Fach“ zu verstehen ist. Nach Adamzik ist ein Fach „ein Gegenstandsbereich, auf dessen (kommunikative) Bearbeitung unter einer bestimmten Perspektive sich in einer Gesellschaft bestimmte Menschen spezialisiert haben“ (Adamzik 2018:95). 
In spezifischen Fachtexten werden Eigenschaften, Ergebnisse, Sachverhalte, Tatbestände, Vorgänge, Prozesse, Ereignisse, Taten, Handlungen, Tätigkeiten, Geschehnisse durch allgemeine Termini u. a. beschrieben. Es wird über fachliche Inhalte kommuniziert, die Bestandteile eines Faches sind.

Unter „Fachsprache“ verstehen wir dann nach Hoffmann „die Gesamtheit aller sprachlichen Mittel, die in einem fachlich begrenzbaren Kommunikationsbereich verwendet werden, um die Verständigung zwischen den in diesem Bereich tätigen Menschen zu gewährleisten“ (Hoffmann 1985:53).

Die Tradition der einzelnen Fachsprachen ist unterschiedlich. Die ältesten fachlichen Quellen gibt es in den Fachgebieten landwirtschaftliche Produktion, Tierhaltung, Jagd, Fischerei und Handwerk, erst später entstehen fachspezifische sprachliche Mittel der Bereiche Geisteswissenschaften, Rechtswesen u. a. (vgl. Adamzik 2018:105, Roelcke 2010:180). Eben die ältesten Berufe sind Handlungsbereiche, bei denen der Beruf und der Alltag schwer zu trennen sind. Zu diesen Berufen gehören u. a. auch Winzer und Bauern. Ziele der Kommunikation unter den Fachleuten sind: sich über Erkenntnisse zu informieren, über theoretische und praktische Probleme, Methoden, Lösungen von Problemen zu diskutieren, kurz gesagt, sich auf einem Fachgebiet zu verständigen. „Eine Fachsprache ist also nicht eine besondere Existenzform von Sprache im Sinne [eines] sprachlichen Systems, sondern eine in fachspezifischer Tätigkeit realisierte Form der Allgemeinsprache" (Bock 1976:11, in: Hoffmann 1985:162).

\section{Weinkunde als Fachgebiet}

„Bei Fächern handelt es sich um soziale Konstrukte, und zwar um solche, die in unserer Bezugswelt innerhalb der Superinstitution Staat explizit institutionalisiert werden. Konkret heißt das, dass man zum Spezialisten bzw. Sachverständiger ausgebildet wird“ (Adamzik 2018:104). Fächer sind heterogen, d. h., sie können unterschiedliche Handlungsbereiche beinhalten. Die Winzersprache z. B.

„bezieht sich allgemein auf die Kommunikation in einem spezifisch menschlichen Praxisbereich, den der Ausbildung, Tätigkeit und Mitteilung im Weinfach. Die historische Winzersprache beruht auf der traditionellen, mit manuellen Techniken betriebenen, vorindustriellen Weinbauarbeit und fast ausschließlich auf mündlicher Kommunikation.“ (Kleiber 1998:1083)

Jedes Fachgebiet besitzt seine sprachlichen Besonderheiten, sei es die Fachsprache der Metallverarbeitung, Fachsprache der Börse, Sprache der Politik, der Physik, medizinische Fachsprache, Verwaltungssprache u. a. Die wichtigsten Wortarten im Hinblick auf die spezifischen fachsprachlichen Mittel innerhalb der Lexik sind Substantive, Adjektive und Verben.

Die Fachsprache über den Wein ist spezifisch. Der Fachwortschatz betrifft mehrere Gebiete:

1. Weinbau: Rebsorten, Bau und Leben eines Weinstockes, physiologische Störungen, die Rebe und ihre Umwelt, Ernährung und Düngung der Rebe, Krankheiten der Rebe, Schädlinge der Rebe / Rebschutz, Weinbau, Neuanlage eines Weinbergs, Rebenveredlung, Rebenzüchtung, Betriebs- und Arbeitswirtschaft, Weinbaurecht u. a.

2. Kellerwirtschaft: Betriebsmittel, Weinbereitung, Weinbehandlung, Fehler und Krankheiten der Weine, Umweltschutzmaßnahmen und Unfallverhütungsvorschriften, Weinbeurteilung, Weinüberwachung u. a.

3. Wein als Produkt: Weintraube, Traubenmost, Gewinnung des Traubenmostes, Gärung, Ausbau der Weine, Anreicherung und Entsäuerung der Traubenmoste und Weine, Behandlung der Traubenmoste und der Weine mit Enzymen, Verschneiden der Weine, Fehler und Krankheiten der Weine, Zusammensetzung und Beurteilung der Weine, Likörweine, Schaumweine, weinhaltige Getränke, Obst- und Beerenweine, Rückstände der Weinbereitung und ihre Verwertung, Untersuchung der Moste und Weine u. a.

Der Fachwortschatz der Diskurse über den Wein beinhaltet u. a. die Terminologie der Botanik: 
Pilze bilden gewöhnlich feine, mit bloßem Auge nicht mehr wahrnehmbare Fäden (Hyphen), die häufig aber in der Masse als zumeist weißes, graues oder braunes Geflecht (Myzel) sichtbar werden.

(Vogt/Götz 1979:258)

der Bodenkunde:

Oberhalb von Tübingen findet sich der Keuper, bei Metzingen Vulkangestein, im Neuffener Tal der Juraboden. In Eßlingen bei Stuttgart und im Remstal kommen der rote, grünliche und bläuliche Keuper vor. In Bad Cannstadt und am Unterlauf der Rems tritt Muschelkalk zutage. Löß und Schotterböden wechseln mit extrem schwerem Lettenkeuper oder Gipskeuper ab.

(Kadisch 1986:24)

Am Westhang des Steigerwaldes dominieren Verwitterungsböden des Keupers mit Mergel und Gips, die würzig-erdhafte, wuchtige und bukettreiche Weine hervorbringen.

(Block/Fritsch/Karow 2000:7)

der Chemie:

Die Maische erhält die $\mathrm{SO}_{2}$-Gabe in Form von Kaliumdisulfit. Kaliumdisulfit gibt es in 10-g-Tabletten oder als Pulver im 50-g-Beutel. [...] Man verwendet 20\%ige Gelatinelösung und 5\%ige schweflige Säurelösung. Bei einer $\mathrm{SO}_{2}$-Gabe auf die Maische von $3 \mathrm{~g} / \mathrm{hl}$ braucht man $60 \mathrm{ml} 5 \%$ $\mathrm{H}_{2} \mathrm{SO}_{2}$-Lösung.

(Meidinger 1989:87)

des Rechtswesens:

Nach $\$ 1$ des Weinwirtschaftsgesetzes (1) bedarf die weinbergmäßige Neuanpflanzung von Weinreben und auch die weinbergmäßige Wiederanpflanzung von Weinreben in gerodeten Weinbergen einer Genehmigung durch die von der Landesregierung bestimmte Behörde. Mit dieser Anbauregelung soll die Erzeugung von minderwertigem Wein verhindert und die Wettbewerbsfähigkeit des deutschen Weinbaus erhöht werden.

(Vogt/Götz 1979:425)

\section{Wein in der Sprache}

Diskurse über Wein sind sehr vielfältig. Was die Wahl der sprachlichen Mittel betrifft, wird sie von vielen Faktoren beeinflusst. Das sind: die Kommunikationsform, das gewählte Medium, der Kommunikationsbereich und die Textsorte, die Intention des Textproduzenten, aber auch andere Aspekte der Kommunikationssituation wie Raum, Zeit und nicht zuletzt die Kommunikationspartner.

\subsection{Wein in der Sprache der Weinbauern}

Die Sprache der Weinbauern wird als Berufssprache und zugleich als Gruppensprache mit Fachausdrücken aus dem Gebiet des Weinbaus, die „Winzerterminologie“ / „Weinbauterminologie“ (vgl. Krämer-Neubert 2008) genannt werden, bezeichnet.

In der Weinbausprache hat das romanische Sprachgut seine Spuren hinterlassen.

„Die noch heute üblichen Benennungen wie Wein (vinum), Winzer (vinitor), Most (mustum), Keller (cellarium) usw., die sämtlich aus der lateinischen Sprache entlehnt sind, können als Beweis dafür angesehen werden, welch großen Einfluss die Römer auf den ältesten deutschen Weinbau hatten.“ (Vogt/Jakob/Lemperle/Weiss 1984:12)

Und so besteht die Rebbauterminologie aus traditionellen Benennungen von Tätigkeiten und Werkzeugen des Ackerbaus und der Pflanzenpflege, die die frühen Siedler in die heutigen Weinbaugebiete mitgebracht haben (vgl. Egli 1982:365). 
Die Sprachwissenschaftler widmen sich auch der jeweiligen mundartlichen Terminologie unterschiedlicher Weinbaugebiete und beschreiben Ergebnisse ihrer wortgeographischen Untersuchungen, wie z. B. zum Deutschwallis (Egli 1982), Burgenland (Resch 1980), in Unterfranken (Krämer-Neubert 2008). Anhand der bisherigen Untersuchungen der Sprache der Weinbauern ist ersichtlich, dass sich der sprachliche Wortschatz verändert hat und sozusagen die Sprache ihr eigenes Leben hatte, und dass der Fachwortschatz der jüngeren Generation nicht mehr lokal gebunden, aber trotzdem immer noch variationsreich ist (vgl. Krämer-Neubert 2008:49). Die dialektologische Fachsprachenforschung ist unter anderem aus dem „Bewahrungs- und Rettungsgedanken“ entstanden (Kleiber 1998:1085), und die zahlreichen Untersuchungen der Winzersprache betrachten die Winzerterminologie als „Quelle der Sprach- und Kulturgeschichte“ (Kleiber 1998:1085). Die Tatsache, dass die gesprochene Sprache der Weinbauern dialektal geprägt ist, hängt damit zusammen, dass für (die) Weinbauern, genauso wie z. B. für (die) Berufsfischer oder andere Handwerker, die an den Boden oder die Landschaft fest gebunden waren, kein Wanderzwang bestand (vgl. Fluck 1996:69). In der modernen Winzersprache kommt es allerdings zu einer Internationalisierung der Sprache durch die gemeinsame Weinmarktorganisation der EU-Länder und zu einem damit verbundenen langsamen Verschwinden von regionalen Varianten innerhalb einer Sprachgemeinschaft. Die Sprache über den Wein besitzt heutzutage, auch dank einer zentralisierten Ausbildung der Winzer, einen überregionalen Charakter.

\subsection{Wein in der Sprache der Fach- und Lehrbücher}

Die Zielgruppe der didaktisierenden Texte bilden Leser, die spezielle Kenntnisse über den Weinbau, die Kellerwirtschaft und den Wein allgemein zu ihrer Ausbildung und Fortbildung benötigen: Winzer, Auszubildende und Besucher von Fachschulen, Absolventen der Meisterausbildung, aber auch Weinfreunde und Praktiker. Beteiligt an diesen Fachbüchern ist meist ein Autorenkreis mit bis zu elf Mitarbeitern. Dass es sich um wichtige und auf diesem Gebiet bedeutende Nachschlagewerke handelt, beweist die Tatsache, dass die untersuchten Bücher immer wieder in einer mehr oder weniger veränderten Form erscheinen. Aber vor allem verändert sich im Zusammenhang mit einer Umstellung im Denken und Handeln sowie einer Umschichtung in der Bedeutung mancher Teilgebiete - aber auch dank der durch Erfolge der Rebenzüchtung gewachsenen Zahl der Rebsorten und Fortschritte in der Entwicklung der Technik und damit auch im praktischen Weinbau - die weinrechtliche Situation (vgl. Vorworte in Vogt/Götz 1979, Vogt/Jakob/Lemperle/Weiss 1984, Kadisch 1986).

Fachtexte tragen Merkmale der Fachsprachlichkeit (vgl. Zhu 2014:238):

1. Sachbezogenheit - Dominanz der deskriptiven, instruktiven und direktiven Funktion der Texte und Verzicht auf emotionale und subjektive Sprachmittel

2. Exaktheit - Termini, Definitionen

3. Sprachökonomie - Informationskomprimierung

4. Anonymisierung - unpersönlicher Stil

Der folgende Text (ein Ausschnitt aus: ,Weinkompendium. Botanik. Sorten. Anbau. Bereitung' von zwei renommierten Autoren) richtet sich an Leser in der beruflichen Ausbildung, die zu Weinexperten ausgebildet werden sollen.

\subsection{Morphologie}

Die Weinrebe ist eine Liane mit sympodial aufgebauten Sprossen. Jedes Sympodialglied endet mit einer Sproßranke, die seitlich abgedrängt wird, während eine Achselknospe die Scheinachse fortsetzt (Abb. 7). Aus Beiknospen der Blattachseln gehen Kurztriebe, „Geiztriebe“ und „Herlinge“, nachgetriebene, meist nicht mehr ausreifende Trauben, hervor, deren Namen sich nach v. Bassermann-Jordan von herb (herw) ableitet. Die Sproßranken entsprechen reduzierten Infloreszenzen, wie schon Albertus Magnus erkannte. 
Im Vordergrund steht die Informationsfunktion, wobei die Informationen in einem neutralen und unpersönlichen Stil präsentiert werden. Vorhanden sind Fachtermini (Liane, sympodial, Sprossen, Sympodialglied, Sproßranke, Achselknospe, Scheinachse, Beiknospen, Blattachseln, Kurztriebe, Geiztriebe, Herlinge u. a.) und Abbildungen. Der Unterschied zwischen gemeinsprachlichen Wörtern und Fachtermini besteht darin, dass die Bedeutung der Termini durch eine exakte Definition festgelegt ist. In Lehrbüchern bilden Fachsprachen das notwendige Instrumentarium zur Stoffbeherrschung (vgl. Fluck 1996:152). Für einen Laien können Fachtexte mit unbekannten Fachtermini eine Kommunikationsbarriere darstellen. In Lehrbüchern werden die schwer verständlichen Inhalte visualisiert und anhand von Abbildungen erklärt:

Abb. 1: Visualisierung von Informationen (Kadisch 1986:27)

\subsection{Wein in populärwissenschaftlichen Texten}

Es handelt sich um popularisierende Ratgebertexte, die vorwiegend für Laien und Hobby-Weinfreunde geschrieben werden:

\section{Bau und Leben des Rebstocks (Anatomie und Physiologie)}

3.1 Organe des Rebstocks

3.1.1 Wurzeln

Die Wurzeln verankern den Rebstock im Boden und nehmen Wasser und Nährstoffe aus dem Boden auf.

Wurzeln entstehen normalerweise aus der Keimlingsanlage eines Samenkorns. Unsere Reben werden aber, außer in der Rebenzüch-
tung, vegetativ (ungeschlechtliche Vermehrung durch Schnittholz oder uber Augensteckling im Gegensatz zur generativen Vermehrung vermehrt. Der Grund hierfür ist darin zu sehen, daß sich bei der Rebe überall an den Sproßtelen, und zwar aus dem Kallusgewebe, Wurzel bilden können. Man spricht hier von Adventiv. wurzeln.

Im deutschen Weinbau werden fast ausschließlich Pfropfreben vermehrt; der Sproß besteht also aus der wurzelbildenden Unterlage und dem Edelreis.

Je nachdem, an welcher Stelle der Unterlage dje Wurzeln herauskommen, spricht man vol Fußwurzeln

- Seitenwurzeln

Tag- oder Tauwurzeln.

Die Fußwurzeln am unteren Ende (Fußende) der Unterlage sind zahlreich und sehr or wünscht, weil sie die Basis für die Ernahrung des Rebstocks bilden.

Die Seitenwurzeln entspringen dem Mittestuck der Unterlage und breiten sich vorwi-

gend in den mittleren und oberen Bodenschich-

ten aus. In begrünten Anlagen können sie sich

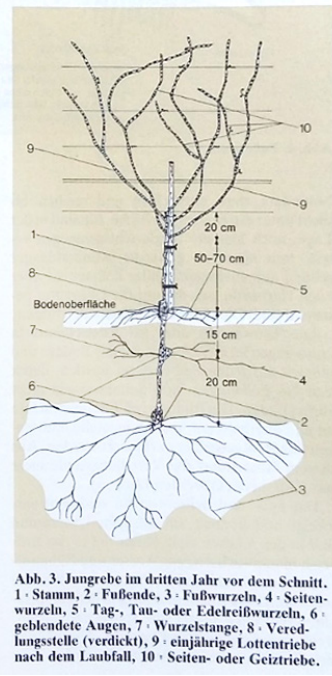

\section{Riesling}

Wein: Grünlich-gelb, elegant mit typischer frischer Säure und nuancenreichem Bukett, bei Vollreife hochbewertete Spätlesen, Auslesen, Beeren- und Trockenbeerenauslesen, bei Edelfäule von besonderer Eigenart, lagerfähig.

Frisch, spritzig, rassig, fruchtig, saftig, kernig, stahlig, nervig, wuchtig und elegant - so lauten je nach Lage, Boden, Jahrgang die Epitheta im Personalausweis des Rieslings. In guten Herbsten übertrifft ihn kein anderes Weißgewächs an Kraft und finessenreichem Spiel. Dann mischen sich Heckenrose und Pfirsich in seinem Bukett, dann halten sich harmonisch voller Körper und geschliffene 
Säure unnachahmlich die Waage. Dann erscheint der sonst so verschrobene Säuerling auf einmal als Mann von Welt.

(Gräter 1990:21)

Ein Sachbuch unterscheidet sich von einem Fachbuch, indem es einen abstrakten Inhalt menschlicher gestaltet. Bei einer gezielten Verschiebung von der Sachorientierung auf die Publikumsorientierung kommt es zur Veranschaulichung der Inhalte, Emotionalisierung und Dynamisierung; Sachtexte sind empfängerorientiert und sollen Interesse wecken (vgl. Pörksen 1980:41, Rykalová 2019:206). Zu den typischen sprachlichen Mitteln gehören Metaphern, wie z. B. elegant, frisch (jung und durch natürliche Kohlensäure lebendig), wuchtig (mit viel Alkohol und Körper), nervig (noch jung und lebhaft), rassig (herzhaft mit gut eingebundenen Fruchtsäuren), Personalausweis des Rieslings, finessenreiches Spiel:

- [...] in guten Herbsten übertrifft ihn kein anderes Weißgewächs [...]

- Dann erscheint der sonst so verschrobene Säuerling auf einmal als Mann von Welt.

\author{
Silvaner, Grüner \\ Wein: Gelbgrünlich bis gelb, vollmundig, milde Säure, zartes Bukett, bei günstiger Witterung auch \\ körperliche Spitzenweine.
}

(Bergner/Lemperle 2001:61)

\title{
Grüner Silvaner
}

Der Silvaner, auch „, Österreicher “ oder ,Fränkischer “ genannt, ist eine in Deutschland weitverbreitete Sorte. Seine Anbauschwerpunkte sind Rheinhessen, die Pfalz, die Nahe und Franken. Der Vorteil dieser Sorte liegt in der hohen Fruchtbarkeit, sie stellt aber hohe Standortansprüche und läßt [sic!] oft in der Reife von Trauben und Holz zu wünschen übrig. Die Traube ist grün, mittelgroß und sehr saftreich, der Wein mundig und mild.

(Vogt/Jakob/Lemperle/Weiss 1984:28)

Die unterscheidenden Merkmale für die Klassifikation der Texte sind das Verhältnis von Information und Redundanz, die Übersichtlichkeit der Anordnung der Information, die Kompliziertheit der Darstellung, der Anteil der optischen Vermittlung von Informationen (vgl. Pörksen 1980:26 f.):

- Der Silvaner, auch „Österreicher“ oder ,Fränkischer“ genannt [...]

- Der Vorteil dieser Sorte liegt in der hohen Fruchtbarkeit, sie stellt aber hohe Standortansprüche und läßt [sic!] oft in der Reife von Trauben und Holz zu wünschen übrig.

\subsection{Wein in der Sprache der Weinkataloge}

Kataloge sind Werbetexte mit einem starken persuasiven Charakter. Kataloge werden für die Zwecke der Vermarktung von Weinprodukten zusammengestellt. Somit handelt es sich um ,sprachliches Handeln mit dem Ziel, Rezipienten mittels verbaler und/oder non-verbaler Kommunikationsmittel von einer Meinung zu überzeugen oder/und sie zu einer bestimmten Handlung zu bewegen“" (Breckle 2012:51) und somit um ein persuasives Handeln.

\section{Würzburger Stein Riesling trocken}

Strahlend und brillant. Typische Aromen von Limette und Pfirsich mit Nuancen von schwarzem Pfeffer. Geradlinige und kühle Stilistik. Sehr dichter, komplexer Steinwein mit langem Nachhall und einer rassigen Säure. Ein Wein, der den Spagat zwischen graziler Anmut und schierer Kraft schafft.

\section{Iphöfer Julius-Echter-Berg Riesling}

Intensiver Duft erinnert an Pfirsich, reife Ananas und Maracuja, samtiges Mundgefühl, mit verführerischer Süße und lebendiger Säure. 


\section{Iphöfer Domina trocken}

Tiefdunkle Farbe im Glas. In der Nase schwarze Kirschen und Brombeeren. Am Gaumen präsentiert er sich sehr würzig mit Aromen von Kakao, Heidelbeeren. Zarte Tannine gepaart mit einer angenehmen Säure.

\section{Würzburger Abtsleite Traminer trocken}

Einnehmende Aromenvielfalt von Rosenblüten und Lavendel über Lychee bis hin zu einem Hauch von Gelbfrucht und Süßholz. Am Gaumen weich, mundfüllend mit gut eingebundenen Holzaromen von Vanille. Ein spannender Wein, der zeigt, was diese alte Traubensorte zu bieten hat. Langes Reifepotential bis zu 10 Jahren möglich.

(URL 1)

In produktbegleitenden Texten werden Begriffe, die positiv konnotiert werden und die das Produkt interessant und attraktiv erscheinen lassen, präsentiert. Produktbeschreibende sprachliche Mittel wie Adjektivattribute und Substantive sind besonders frequent. Wertende (qualitative und quantitative) Adjektive rufen positive Konnotationen hervor (z. B. strahlend, brillant, geradlinig, dicht, komplex, samtig, würzig, angenehm), die in Verbindung mit dem nachgestellten Nomen besondere Effekte erzeugen (z. B. komplexer Steinwein, langer Nachhall, rassige Säure, grazile Anmut, schiere Kraft, intensiver Duft, zarte Tannine). Für diese Texte ist typisch, dass die Informationsvermittlung vorwiegend mittels nominaler Wortverbindungen mit einer elliptischen Prädikation erfolgt. Somit werden die Eigenschaften des Weines in den Vordergrund gestellt. Typisch für die Weinsprache sind Substantive, die Sinnesempfindungen, Pflanzen und Früchte benennen (z. B. Limette, Pfirsich, schwarzer Pfeffer, Ananas, Maracuja, schwarze Kirschen, Brombeeren, Kakao, Heidelbeeren). Es handelt sich um Metonymien (z. B. In der Nase schwarze Kirschen und Brombeeren.) und Vergleiche (z. B. Intensiver Duft erinnert an Pfirsich, reife Ananas und Maracuja.). Für die Weinsprache typische Begriffe kommen in Form von Substantiven vor (z. B. Aromen, Nuancen, Stilistik, Säure, Tannine) sowie in musterhaften Formulierungen (z. B. in der Nase, am Gaumen / sich am Gaumen präsentieren).

Für Kataloge und Werbetexte ist eine bildhafte Ausdrucksweise typisch, die u. a. durch metaphorische Benennungen erreicht wird:

- Geradlinige und kühle Stilistik.

- Sehr dichter, komplexer Steinwein mit langem Nachhall und einer rassigen Säure.

- [...] samtiges Mundgefühl, mit verführerischer Süße und lebendiger Säure.

- Ein Wein, der den Spagat zwischen graziler Anmut und schierer Kraft schafft.

\subsection{Wein in der Sprache der Weinverkostung}

Die große geschmackliche Vielfalt, die der Wein bietet, ist faszinierend.

„Kein Wein ist dem anderen gleich. Von allen Nahrungs- und Genussmitteln ist er wohl das vielfältigste. Er unterscheidet sich nach Rebsorten und Lagen, Bodenbeschaffenheit und Jahrgängen, Lese im Weinberg und Ausbau im Keller, aber auch nach Lagerung und Alter, Trinktemperatur und Zusammenklang mit Speisen." (Althaus 2008:7)

Obwohl der folgende Text aus einer der untersuchten Quellen stammt, könnte er als Beispiel für einen Kommentar bei der Weinverkostung dienen:

Traminer: Er läßt [sic!] sich zu gerne von der Sonne verwöhnen, kuschelt in den wärmsten Lagen, braucht tiefgründige Böden und entfaltet seinen Charme, sein delikates Aroma nur, wenn er spät, kurz vor dem Riesling, gelesen wird.

Dafür betört der bernsteingelb im Glas schimmernde Wein dann mit einem großartigen Heckenrosenbukett, in dem nach Meinung witternder Prüfnasen zuweilen auch ein Hauch Veilchen grüßen läßt [sic!], eine Prise Vanille durchdringt.

(Gräter 1990:38) 
Eine bildhafte Sprache ruft bestimmte Assoziationen hervor, welche wiederum (in unserem Kontext) positive Emotionen, die eine persuasive Wirkung haben, beinhalten. Ungewöhnliche und/oder emotional besetzte Ausdrücke wecken die Aufmerksamkeit der Rezipienten und unterstützen die Persuasion der Texte.

Bilder sowie Personifizierungen bilden den Faden zu eigenen Erlebnissen und aktivieren die damit verbundenen Emotionen:

- sich gerne von der Sonne verwöhnen lassen

- in den wärmsten Lagen kuscheln

- seinen Charme entfalten

- grüßen lassen

Sorgfältig gewählte Adjektiv- und Partizipialattribute wirken einladend:

- delikates Aroma / großartiges Heckenrosenbukett / bernsteingelb schimmernde genauso wie die für die Weinbeschreibung typischen Aroma- und Geschmacksbezeichnungen:

\section{- Heckenrosenbukett / ein Hauch Veilchen / eine Prise Vanille}

Die Kommentare bei Weinverkostungen für eine breite Öffentlichkeit „dienen nur teilweise der Information, manche sind rein dekorativ" (Althaus 2008:8). Damit soll ausgedrückt werden, dass diese Kommentare, wie auch produktbegleitende Texte (siehe oben), den Wein bei der Weinverkostung attraktiv und interessant erscheinen lassen sollen. Exakt formulierte reine Fakten sind weniger wichtig als interessant, witzig und originell formulierte Informationen sowie eine gemütliche Atmosphäre.

Eine andere Ausdrucksweise gehört allerdings zur Sprache der Degustation durch Experten (vgl. Althaus 2008, Meidinger 1989). Die Weinexperten äußern sich sehr exakt über die Klarheit, Farbe und Reinheit des Weines, über den Geruch und über den Geschmack und den nachwirkenden Eindruck im Mund. Erwähnt werden Fruchtaromen, Gewürznoten oder eine holzige Note; beschrieben wird, wie sich der aromatische Eindruck der Nase am Gaumen fortsetzt, welcher Eindruck sich auf die Zunge legt, ob der Wein frisch und / oder mineralisch wirkt und vielleicht an Eleganz gewinnt.

\subsection{Wein im Alltagshandeln}

Zu den ältesten Diskursen über Wein gehören ohne Zweifel Weinlieder, Weinsprüche, unterschiedliche Zitate, Sprichwörter und geflügelte Worte. Sie haben vor allem einen unterhaltenden Charakter:

- Das Leben ist viel zu kurz, um schlechten Wein zu trinken. - Johann Wolfgang von Goethe

- Regen lässt das Gras wachsen, Wein das Gespräch.

- In vino veritas.

- Jetzt schwingen wir den Hut,

- der Wein, der war so gut. (Gräter 1990:46)

Gesprächen beim Weinkauf, Weinliedern, Weinsprüchen, Werbung u. a. begegnen wir im alltäglichen Leben. Beim Alltagshandeln entstehen einerseits Texte, die primär einen unterhaltenden Charakter haben, wie z. B. Geschichten und Anekdoten für und über die Weinliebhaber, auf der anderen Seite werbende Texte, die den Wein als Produkt begleiten und seine Attraktivität erhöhen sollen. Eins haben beide Textsammlungen gemeinsam: Das Vokabular, das zur Beschreibung von Wein dient, ist sehr umfangreich und vielfältig. Der Wein wird gelobt, genossen, besungen. Die verwendeten sprachlichen Mittel rufen positive Emotionen hervor. 


\section{Fazit}

Diskurse über den Wein stellen einen sehr interessanten und vielfältigen Untersuchungsgegenstand dar. Wein wird in allen Kommunikationsbereichen thematisiert - sei es im Kommunikationsbereich Fachkommunikation, schöne Literatur, Alltagsverkehr oder Massenmedien und offizieller Verkehr (die in dieser Studie nicht angesprochen wurden). Im Kommunikationsbereich Belletristik wird der Wein in Liedern, Gedichten, Erzählungen u. a. besungen, im Kommunikationsbereich Fachkommunikation finden wir Fachtexte für und/oder von Fachexperten, im alltäglichen Umgang wird der Wein meistens im Zusammenhang mit einer schönen Atmosphäre, Genuss und Gemütlichkeit erwähnt usw.

Die Sprache der Weinkenner finden wir vor allem in Fachbüchern. „Das Fachbuch ist für Spezialisten bestimmt, für den Lernenden oder den Berufstätigen, die sich mit dem Stoff auseinandersetzen müssen [...]. Beim Sachbuch ist es umgekehrt: Wer danach greift, tut es freiwillig...Und dabei tritt das Sachbuch in Konkurrenz mit vielerlei Unternehmen der Freizeitindustrie [...]. Das moderne Sachbuch folgt der Strategie der Werbung [...]“ (Franke 1980:45).

Fachwissen wird auch in Sachbüchern vermittelt, die primär als Kommunikationsmittel zwischen Fachleuten und Laien dienen. „Fachtermini sollen in diesen Texten keine emotionalen, distanzierenden Wirkungen beim Rezipienten hervorrufen, sondern ihm ein Minimum an Kenntnissen spezialsprachlicher Terminologie vermitteln, die meist ausführlich, variantenreich und anschaulich erläutert wird“" (Fluck 1996:174). Dabei verzichtet der Text auf fachliche Exaktheit, zugunsten einer besseren Verständlichkeit wird die Sprache mittels metaphorischer Vergleiche visualisiert, typisch ist auch sprachliche Redundanz.

Produktbegleitende Texte, wie Weinbeschreibungen auf unterschiedlichen Webseiten und auch Kommentare bei der Weinverkostung, beinhalten zwar Fachtermini; typisch sind aber vor allem charakterisierende Adjektivattribute und eine bildhafte Sprache, die bestimmte Assoziationen hervorruft. Ungewöhnliche und/oder emotional besetzte Ausdrücke wecken die Aufmerksamkeit der Rezipienten (der potenziellen Käufer). Werbesprache bedient sich auch anderer Varietäten und arbeitet sehr oft mit Wortspielen und okkasionellen Bildungen, um bestimmte Assoziationen hervorzurufen. Verwendet werden Begriffe, die positiv konnotiert werden und die das Produkt interessant und attraktiv erscheinen lassen. Werbende Texte haben einen starken persuasiven Charakter.

\section{Literaturverzeichnis}

\section{Primärliteratur:}

Althaus, Hans Peter (2008): Kleines Wörterbuch der Weinsprache. München.

Bergner, Karl-Gustav / Lemperle, Edmund (2001): Weinkompendium. Botanik. Sorten. Anbau. Bereitung. 3. Aufl. Stuttgart; Leipzig.

Block, Detlev / Fritsch, Jürgen / Karow, Antonia (2000): Auf den Spuren des Weines. Eine kulinarische Weinreise. Gütersloh.

Broadbent, Michael J. (1979): Weine prüfen, kennen, geniessen. 2. Aufl. Luzern; Stuttgart.

GRÄTER, Carlheinz (1990): Frankens Reben im Portrait. Würzburg: Mainpresse.

Jacques' Neuvorstellungen. April 2019 (2019). Würzburg. (Prospekt)

KADISCH, Erwin (1986): Der Winzer 1. Weinbau. Stuttgart.

MeIDINGER, Friedrich (1989): Der Winzer 2. Kellerwirtschaft. 2. Aufl. Stuttgart.

PrIEWE, Jens (2001): Wein. Die praktische Schule. München. 3. Aufl.

RobInson, Jancis (2017): Weinexperte in 24 Stunden. Von der angesehensten Weinexpertin der Welt. München.

Sommerzeit ist Weinzeit (2019). Würzburg. (Prospekt)

Vogt, Ernst / GöTz, Bruno (1979): Weinbau. Ein Lehr- und Handbuch für Praxis und Schule. 6. Aufl. Stuttgart.

Vogt, Ernst / JAKob, Ludwig / LemPerle, Edmund / Weiss, Erich (1984): Der Wein. 9. Aufl. Stuttgart. 


\section{Sekundärliteratur:}

AdAMzIK, Kirsten (2018): Fachsprachen. Die Konstruktion von Welten. Tübingen.

BRECKLE, Margit (2012): „Frisch durch den Sommer“: Exemplarische Analyse persuasiver Strategien in Titelbeiträgen einer Kundenzeitschrift der Apothekenbranche. In: IAKUSHEVICH, Marina / ARnING, Astrid (Hrsg.): Strategien persuasiver Kommunikation. Hamburg, S. 49-69.

EgLI, Alfred (1982): Weinbau im Deutschwallis. Struktur. Wortschatz. Sprachgeographie. Frauenfeld. FLuCK, Hans-Rüdiger (1996): Fachsprachen. 5. Aufl. Tübingen; Basel.

FrANKe, Herbert W. (1980): Das naturwissenschaftlich-technische Sachbuch. In: Zeitschrift für Literaturwissenschaft und Linguistik. Jg. 10/1980, Heft 40, S. 44-51.

HofFMAnN, Lothar(1985): Kommunikationsmittel Fachsprache. Eine Einführung. 2. Aufl. Tübingen. KLEIBER, Wolfgang (1998): Die Fachsprache der Winzer unter besonderer Berücksichtigung des Rhein-Mosel-Gebiets. In: HSK 14.1 Fachsprachen Languages for Special Purposes. Berlin; New York, S. 1083-1092.

KRÄMER-NeUbERT, Sabine (2008): Expertendialekt - Zum Fachwortschatz der Winzer in Unterfranken. In: Schriften zur Weingeschichte, Nr. 162, Wiesbaden, S. 41-51.

PöRKSEN, Uwe (1980): Populäre Sachprosa und naturwissenschaftliche Sprache. In: Zeitschrift für Literaturwissenschaft und Linguistik. Jg. 10/1980, Heft 40, S. 25-43.

Resch, Gerhard (1980): Die Weinbauterminologie des Burgenlandes. Eine wortgeographische Untersuchung, ausgehend von der Weinbaugemeinde Gols. Wien.

RoElcKe, Thorsten (2010): Fachsprachen. 3. Aufl. Berlin.

RYKalová, Gabriela (2019): Die Rolle der Termini in Kapitelüberschriften auf unterschiedlichen Ebenen der Fachlichkeit. In: VaŇKové, Lenka (Hrsg.): Fachlichkeit und Fachsprachlichkeit in varianten Kontexten. Berlin, S. 197-212.

ZHU, Jianhua (2014): Fachstile und Fachkulturen in der globalisierten Welt. In: BuRKHARDT, Armin / ZhaO, Jin / ZHU, Jianhua (Hrsg.): Alltags- und Fachkommunikation in der globalisierten Welt. Frankfurt a. M., S. 237-243.

\section{Elektronische Quellen:}

URL 1: https://www.weingut-juliusspital.de. [01.09.2019].

Dieser Beitrag entstand mit Unterstützung des Projektes der Schlesischen Universität in Opava SGS/1/2020, Perspektivy textové analýzy ve 21. stoleti '. 